\title{
Effect of Cognitive Impairment on Driving-Relevant Cognition in Older Persons
}

\author{
Rahel Bieri ${ }^{1}$, Michael Jäger ${ }^{1}$, Nora Bethencourt ${ }^{1}$, Urs Peter Mosimann ${ }^{1,2}$, René Martin Müri ${ }^{1,3}$ and Tobias Nef ${ }^{1,4}$ \\ 1. Gerontechnology \& Rehabilitation Group, University of Bern, Bern 3010, Switzerland \\ 2. Department of Old Age Psychiatry, University Hospital of Psychiatry, University of Bern, Bern 3010, Switzerland \\ 3. Departments of Neurology and Clinical Research, University Hospital Inselspital, University of Bern, Bern 3010, Switzerland \\ 4. ARTORG Center for Biomedical Engineering Research, University of Bern, Bern 3010, Switzerland
}

\begin{abstract}
Intact cognitive abilities are fundamental for driving. Driving-relevant cognition may be affected in older drivers due to aging or cognitive impairment. The aim of this study was to investigate the effects of cognitive impairment on driving-relevant cognition in older persons. Performance in selective and divided attention, eye-hand-coordination, executive functions and the ability to regulate distance and speed of 18 older persons with CI-Group (cognitive impairment group) was compared to performance of older control group (18 age and gender-matched cognitively normal subjects) and young control group (18 gender-matched young subjects). The CI-Group showed poorer performance than the other two control groups in all cognitive tasks (significance level $(p)<$ 0.001 , effect size (partial $\left.\eta^{2}\right)=0.63$ ). Differences between cognitively impaired and cognitively normal subjects were still significant after controlling for age (effect sizes from 0.14 to 0.28 ). Dual tasking affected performance of cognitively impaired subjects more than performance of the other two groups $\left(p=0.016\right.$, partial $\left.\eta^{2}=0.14\right)$. Results show that cognitive impairment has age-independent detrimental effects on selective and divided attention, eye-hand-coordination, executive functions and the ability to regulate distance and speed. Largest effect sizes are found for reaction times in attention tasks.
\end{abstract}

Key words: Cognitive impairment, aging, driving, cognition, cognitive assessment, age differences.

\section{Introduction}

Driving a car is a complex instrumental activity of daily living, which requires intact cognitive abilities [1]. Cognitive skills fundamental for driving are executive skills, attention, visual scanning and processing [2, 3]. They are frequently affected in cognitively impaired older persons [4]. The extent and pattern of driving-relevant cognitive deficits in cognitively impaired older drivers have not been fully clarified yet [5], especially the ability to perform multiple tasks at the same time, i.e., to divide one's attention, seems to be crucial for driving [6] and even a relatively mild impairment may contribute to impaired driving [7-9]. It is well known that the ability to perform multiple tasks at the same time

Corresponding author: Tobias Nef, Dr., Professor, research fields: gerontechnology, rehabilitation and biomedical engineering. E-mail: tobias.nef@artorg.unibe.ch. decreases over the life span $[10,11]$ and that divided attention may be affected even in very early stages of cognitive impairment [12].

In the present study, we investigated how cognitive impairment affects driving-relevant skills in older drivers. For this purpose, a computer-based test system was used to measure selective and divided attention, eye-hand-coordination, executive functions, and distance and speed regulation [13]. A group of cognitively impaired older subjects as well as a group of cognitively normal older subjects and a group of young subjects completed in the tasks. Cognitive impairment was defined as a score in the MoCA (Montreal cognitive assessment) [14] below 26. Performance of cognitively impaired older subjects was then compared to performance of cognitively normal older subjects. In order to identify age-independent effects of cognitive impairment, the 
two older groups were matched for age and age was included in analyses as a covariate.

\section{Methods}

\subsection{Participants}

Three groups of participants were included in this study (Table 1). The first group (CI-Group) consisted of 18 older adults with cognitive impairment (MoCA score between 19 and 26; eight women, 10 men; mean age $=73.1$ years, $S D=7.3$; age range $65-87$ years). As a second group (older control group), an age and gender matched group of 18 cognitively normal older participants was selected (MoCA score $\geq 26$; eight women, 10 men; mean age $=73.3$ years, $S D=7.1$ years; age range 65-87). Finally, a gender matched group of 18 young participants (young control group) was included (eight females, 10 males; mean age $=$ 29.2; $S D=3.3$, age range 24-35 years). Participants were recruited from Departments of Neurology and Old Age Psychiatry, the Memory Clinic of the University Hospital Bern and with insertions in local newspapers. All subjects were required to have a corrected far visual acuity of 0.5 or higher and a near visual acuity of 0.8 or higher. The study was carried out in accordance with the Declaration of Helsinki and was approved by the local ethics board of the Canton Bern. Written informed consent was obtained from all participants prior to inclusion. No compensation was provided for participation.

\subsection{Apparatus and Materials}

\subsubsection{Demographics}

Demographic data were collected in a structured interview. Near visual acuity (test distance $40 \mathrm{~cm}$ ) and far visual acuity (test distance $5 \mathrm{~m}$ ) were measured with participants wearing glasses if needed, using Landolt C or Snellen charts in decimals [15].

Four paper and pencil screening tests for cognitive functioning were used: the MoCA [14], the TMT (trail making test) versions $\mathrm{A}$ and $\mathrm{B}$ [16], and the clock drawing test [17]. The MoCA screens for global cognitive functioning (executive functions; visual-constructional abilities; short-term memory; language; attention, concentration, and working memory; and temporal and spatial orientation) and takes about 10-15 min. The scoring ranges from 0 to 30 points [14]. The TMT A is a quick test for visual attention and takes about $3 \mathrm{~min}$. The TMT B measures executive functions and takes 3-5 $\mathrm{min}$ [16]. The result in TMT A and B corresponds to the time (in seconds) needed to complete the test. The clock drawing test measures visual-constructional abilities, abstract thinking and executive functions and takes up to 5 min. The scoring ranges from 1 to 7 points [17].

Table 1 Means and SD (standard deviations) for demographics of the study sample.

\begin{tabular}{|c|c|c|c|c|}
\hline Demographic variable & CI-Group $(N=18)$ & $\begin{array}{l}\text { Older control group } \\
(N=18)\end{array}$ & $\begin{array}{l}\text { Young control group } \\
(N=18)\end{array}$ & Significance \\
\hline Age (years) $(S D)$ & $73.1(7.3)$ & $73.3(7.1)$ & $29.2(3.3)$ & - \\
\hline Males/females & $10 / 8$ & $10 / 8$ & $10 / 8$ & - \\
\hline $\begin{array}{l}\text { Far visual acuity } \\
\text { (decimals) }(S D)\end{array}$ & $0.52(0.2)$ & $0.65(0.3)$ & $1.18(0.2)$ & - \\
\hline $\begin{array}{l}\text { Near visual acuity } \\
\text { (decimals) }(S D)\end{array}$ & $0.95(0.1)$ & $1.0(0.0)$ & $1.0(0.0)$ & - \\
\hline $\mathrm{MoCA}$ & $21.1(3.2)$ & $28.3(1.9)$ & $29.8(0.5)$ & $\begin{array}{l}F(1.4,24.4)=91.8, p<0.001 \\
\text { partial } \eta^{2}=0.84\end{array}$ \\
\hline TMT A & $52.4(32.0)$ & $35.0(12.7)$ & $17.3(5.33)$ & $\begin{array}{l}F(1.2,20.3)=12.7, p=0.001 \\
\text { partial } \eta^{2}=0.43\end{array}$ \\
\hline TMT B & $234.3(287.3)$ & $86.5(37.3)$ & $41.9(18.7)$ & $\begin{array}{l}F(1.3,19.1)=13.9, p=0.001 \\
\text { partial } \eta^{2}=0.48\end{array}$ \\
\hline Clock drawing test & $4.6(1.7)$ & $5.6(2.3)$ & $6.8(0.7)$ & $\begin{array}{l}F(1.5,25.5)=8.5 \\
p=0.003, \text { partial } \eta^{2}=0.33\end{array}$ \\
\hline
\end{tabular}




\subsubsection{BCST (Bern Cognitive Screening Test)}

The BCST [13] is a novel computer-based test system consisting of five subtests to assess selective and divided attention, eye-hand-coordination, executive functions, and distance and speed regulation. The subjects were seated at a distance of $50 \mathrm{~cm}$ from a 24 inches screen (width $520 \mathrm{~mm}$, height $325 \mathrm{~mm}$, refresh rate $60 \mathrm{~Hz}$; resolution 1,680 × 1,050 pixels) connected to a desktop computer with Windows 7 (Microsoft Inc.), on which the tests were presented. To measure the subjects' responses, a commercially available steering-wheel (Logitech Driving Force GT) with foot-pedal was used (Fig. 1). Test administration took about $15 \mathrm{~min}$. Tests were realized in MATLAB ${ }^{\circledR}$ R2007b (The MathWorks Inc.). The refresh rate of the image presentation as well as of the measurement of the position of the steering-wheel and foot pedal was $30 \mathrm{~Hz}$.

Each task was explained to participants orally and they could train until they were able to carry it out correctly. To quantify performance, in all subtests the number of errors was assessed; in subtests 1,3 , and 4 in addition to errors reaction times were measured. The details of the test and test psychometrics have been introduced by Bieri et al. [13].

\subsection{Statistical Analyses}

SPSS Software (version 20) was used for statistical analysis. For Tests 1, 3 and 4 of the BCST, the total number of errors was obtained by adding missed targets and false positive responses. In order to assign equal weight to each test of the BCST, a global score for BCST performance was calculated by transforming the individual raw scores in the five subtests into percentile ranks and then calculating the median value.

In order to correct for multiple testing, whenever possible multivariate analyses were performed. Variables with interval scale were analyzed using MANOVA (multivariate analysis of variance) or a Friedman's test if conditions for the MANOVA were

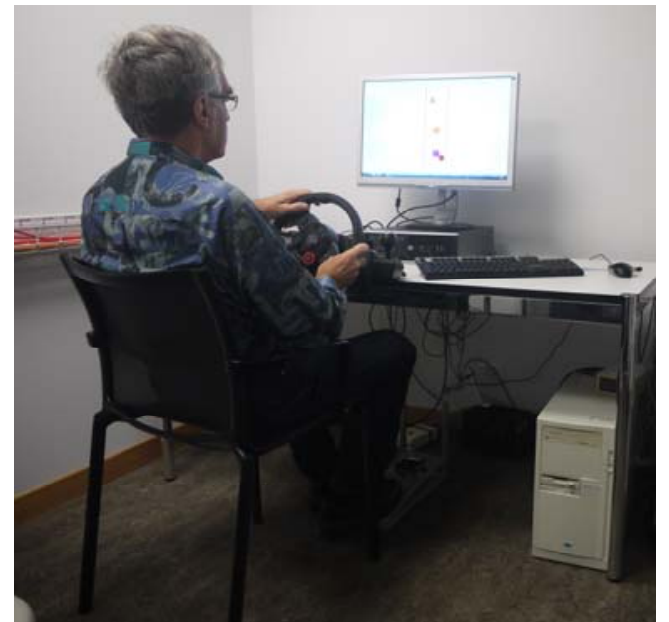

Fig. 1 Setup of the BCST with screen, steering-wheel, and foot-pedal.

not met. Sphericity was tested with a Mauchly-Test and homogeneity for between-subjects analyses with Box's $\mathrm{M}$ test. If the sphericity condition was violated, a Greenhouse-Geisser correction was applied. A $p$-value $<0.5$ was considered as to indicate statistical significance. The effect of age and cognitive status on performance in the BCST was investigated with a two way univariate ANOVA (analysis of variance). In order to explore the effect of specific factors after controlling for covariates, MANCOVA (multivariate analyses of covariance) were calculated. Differential effects of dual tasking on performance of the three groups were investigated using a mixed-model ANOVA of group (CI-Group, older control group and young control group) $\times$ task condition (single task, dual task). To clarify significant interactions, post-hoc tests with a Bonferroni correction were calculated. Homogeneity of variance was tested with a Levene test. Reported $p$-values are two-sided. Appropriate measures for effect sizes are reported. In the interpretation of effect sizes, Cohen's [18] guidelines were followed: $0.20=$ small effect size, $0.50=$ medium effect size, $0.80=$ large effect size.

\section{Results}

\subsection{Demographic Data}

A total number of 54 participants, divided into three 
age and/or gender-matched groups, were included in the study: 18 cognitively impaired older participants (CI-Group), 18 cognitively normal older participants (older control group) and 18 young participants (young control group). As it is shown in Table 1, a one-way MANOVA for related samples revealed significant global group differences in all paper and pencil cognitive tests (Pillai-Spur $=0.88, F(8,64)=$ 60.0, $p<0.001$, partial $\eta^{2}=0.82$ ). Planned contrasts show that that the older control group outperformed the CI-Group in MoCA $(F(1,17)=72.2, p<0.001$, partial $\left.\eta^{2}=81\right)$ and TMT B $(F(1,15)=5.1, p=0.038$, partial $\left.\eta^{2}=0.26\right)$, but not in TMT A $(F(1,17)=4.1, p$ $=0.059$, partial $\left.\eta^{2}=0.19\right)$ and clock drawing test $(F(1$, 17) $=2.3, p=0.146$, partial $\eta^{2}=0.12$ ). Effect sizes are large for the MoCA, which was used as grouping variable, and medium or small for the other paper and pencil tests. Furthermore, the young control group performed significantly better than both older groups in all paper and pencil tests (all $p<0.05$, partial $\eta^{2}$ between 0.21 and 0.90 ).

\subsection{Bern Cognitive Screening Test}

A one-way MANOVA for related samples revealed significantly different performance in the BCST for the three groups (Pillai-Spur $=1.3, F(20,52)=4.3, p$ $<0.001$, partial $\eta^{2}=0.63$ ). Subsequent within-subjects analyses on the level of single performance variables (single subtests and global score) showed that the three groups differ significantly in all of them (Table 2) with medium to large effect sizes (partial $\eta^{2}$ between 0.31 and 0.86 ).

Contrasts between the CI-Group and the other two control groups are presented in Table 3. First, the two older groups were compared. Forty-four percent of the total variance in the BCST between the CI-Group and the older control group is explained by the grouping variable (i.e., cognitive status), which corresponds to a

Table 2 Means, SD and group differences for performance in the BCST.

\begin{tabular}{|c|c|c|c|c|c|}
\hline Test & Performance variable & CI-Group $(N=18)$ & $\begin{array}{l}\text { Older control } \\
\text { group }(N=18)\end{array}$ & $\begin{array}{l}\text { Young control } \\
\text { group }(N=18)\end{array}$ & Significance \\
\hline $\begin{array}{l}\text { BCST total } \\
\text { score }\end{array}$ & $\begin{array}{l}\text { Median percentile } \\
\text { rank test } 1-5\end{array}$ & $75.4(15.4)$ & $59.1(14.7)$ & $22.2(10.3)$ & $\begin{array}{l}F(2,34)=99.9, p<0.001, \\
\text { partial } \eta^{2}=0.86\end{array}$ \\
\hline \multirow{2}{*}{ Selective attention } & $\begin{array}{l}\text { Number of errors } \\
\text { (periphery) (SD) }\end{array}$ & $4.5(5.6)$ & $2.1(1.3)$ & $0.3(0.6)$ & \multirow{2}{*}{$\begin{array}{l}F(1.1,18.5)=7.6 \\
p=0.011 \\
\text { partial } \eta^{2}=0.31 \\
F(1.3,21.4)=23.3 \\
p<0.001 \\
\text { partial } \eta^{2}=0.58\end{array}$} \\
\hline & $\begin{array}{l}\text { Reaction time correct } \\
\text { responses }(\mathrm{ms})(\mathrm{SD})\end{array}$ & $718.3(127.4)$ & $606.4(60.6)$ & $524.1(44.1)$ & \\
\hline Eye-hand-coordination & $\begin{array}{l}\text { Number of collisions } \\
\text { (steering-task) (SD) }\end{array}$ & $47.5(25.9)$ & $30.6(15.5)$ & $10.1(6.4)$ & $\begin{array}{l}F(2,34)=24.1 \\
p<0.001 \\
\text { partial } \eta^{2}=0.59\end{array}$ \\
\hline \multirow{3}{*}{$\begin{array}{l}\text { Divided } \\
\text { attention }\end{array}$} & $\begin{array}{l}\text { Number of errors } \\
\text { (periphery) (SD) }\end{array}$ & $17.5(11.3)$ & $10.9(4.9)$ & $6.2(1.0)$ & \multirow{3}{*}{$\begin{array}{l}F(1.2,20.6)=10.9, \\
p=0.002, \\
\text { partial } \eta^{2}=0.39 \\
F(2,34)=37.1 \\
p<0.001 \\
\text { partial } \eta^{2}=0.69 \\
F(2,34)=23.4 \\
p<0.001 \\
\text { partial } \eta^{2}=0.58\end{array}$} \\
\hline & $\begin{array}{l}\text { Reaction time correct } \\
\text { responses }(\mathrm{ms})(\mathrm{SD})\end{array}$ & $734.0(89.8)$ & $655.8(86.0)$ & $548.2(49.1)$ & \\
\hline & $\begin{array}{l}\text { Number of collisions } \\
\text { (steering-task) (SD) }\end{array}$ & $49.2(28.5)$ & $30.1(16.2)$ & $10.3(5.0)$ & \\
\hline \multirow{2}{*}{ Executive functions } & $\begin{array}{l}\text { Number of errors (ms) } \\
\text { (SD) }\end{array}$ & $11.0(8.9)$ & $3.5(2.6)$ & $1.2(1.3)$ & \multirow{2}{*}{$\begin{array}{l}F(1.1,18.7)=15.4 \\
p=0.001 \\
\text { partial } \eta^{2}=0.48 \\
F(2,34)=12.6 \\
p<0.001 \\
\text { partial } \eta^{2}=0.43\end{array}$} \\
\hline & $\begin{array}{l}\text { Reaction time correct } \\
\text { responses (ms) (SD) }\end{array}$ & $766.5(141.3)$ & $645.1(124.8)$ & $576.0(90.0)$ & \\
\hline $\begin{array}{l}\text { Distance \& speed } \\
\text { regulation }\end{array}$ & $\begin{array}{l}\text { Number of collisions } \\
\text { (SD) }\end{array}$ & $14.7(6.0)$ & $11.0(2.4)$ & $5.7(2.3)$ & $\begin{array}{l}F(1.3,22)=22.8, \\
p<0.001, \\
\text { partial } \eta^{2}=0.57\end{array}$ \\
\hline
\end{tabular}


Table 3 Planned contrasts in BCST-performance between the CI-Group and the two control groups.

\begin{tabular}{|c|c|c|c|}
\hline Test & Performance variable & $\begin{array}{l}\text { CI-Group compared to older control } \\
\text { group }\end{array}$ & $\begin{array}{l}\text { CI-Group compared to young } \\
\text { control group }\end{array}$ \\
\hline BCST total score & $\begin{array}{l}\text { Median percentile rank tests } \\
1-5\end{array}$ & $\begin{array}{l}F(1,17)=13.3, p=0.002 \\
\text { partial } \eta^{2}=0.44\end{array}$ & $\begin{array}{l}F(1,17)=201.6, p<0.001, \text { partial } \\
\eta^{2}=0.92\end{array}$ \\
\hline \multirow{2}{*}{ Selective attention } & Number of errors & $\begin{array}{l}F(1,17)=3.4, p=0.084 \\
\text { partial } \eta^{2}=0.17\end{array}$ & $\begin{array}{l}F(1,17)=10.9, p=0.004 \\
\text { partial } \eta^{2}=0.39\end{array}$ \\
\hline & $\begin{array}{l}\text { Reaction time correct } \\
\text { responses }\end{array}$ & $\begin{array}{l}F(1,17)=10.6, p=0.005 \\
\text { partial } \eta^{2}=0.38\end{array}$ & $\begin{array}{l}F(1,17)=35.2, p<0.001 \\
\text { partial } \eta^{2}=0.67\end{array}$ \\
\hline Eye-hand-coordination & Number of collisions & $\begin{array}{l}F(1,17)=7.4, p=0.014 \\
\text { partial } \eta^{2}=0.30\end{array}$ & $\begin{array}{l}F(1,17)=40.8, p<0.001 \\
\text { partial } \eta^{2}=0.71\end{array}$ \\
\hline \multirow{3}{*}{ Divided attention } & Number of errors & $\begin{array}{l}F(1,17)=4.8, p=0.042 \\
\text { partial } \eta^{2}=0.22\end{array}$ & $\begin{array}{l}F(1,17)=16.9, p=0.001 \\
\text { partial } \eta^{2}=0.50\end{array}$ \\
\hline & $\begin{array}{l}\text { Reaction time correct } \\
\text { responses }\end{array}$ & $\begin{array}{l}F(1,17)=12.0, p=0.003 \\
\text { partial } \eta^{2}=0.41\end{array}$ & $\begin{array}{l}F(1,17)=68.2, p<0.001 \\
\text { partial } \eta^{2}=0.80\end{array}$ \\
\hline & Number of collisions & $\begin{array}{l}F(1,17)=9.7, p=0.006 \\
\text { partial } \eta^{2}=0.36\end{array}$ & $\begin{array}{l}F(1,17)=34.2, p<0.001 \\
\text { partial } \eta^{2}=0.67\end{array}$ \\
\hline \multirow{2}{*}{ Executive functions } & Number of errors & $\begin{array}{l}F(1,17)=10.7, p=0.005 \\
\text { partial } \eta^{2}=0.37\end{array}$ & $\begin{array}{l}F(1,17)=20.8, p<0.001 \\
\text { partial } \eta^{2}=0.55\end{array}$ \\
\hline & $\begin{array}{l}\text { Reaction time correct } \\
\text { responses }\end{array}$ & $\begin{array}{l}F(1,17)=7.8, p=0.012 \\
\text { partial } \eta^{2}=0.32\end{array}$ & $\begin{array}{l}F(1,17)=27.2, p<0.001 \\
\text { partial } \eta^{2}=0.62\end{array}$ \\
\hline $\begin{array}{l}\text { Distance \& speed } \\
\text { regulation }\end{array}$ & Number of collisions & $\begin{array}{l}F(1,17)=5.9, p=0.026 \\
\text { partial } \eta^{2}=0.26\end{array}$ & $\begin{array}{l}F(1,17)=31.0, p<0.001, \\
\text { partial } \eta^{2}=0.65\end{array}$ \\
\hline
\end{tabular}

medium effect size. Furthermore, in all subtests cognitively normal older persons outperform cognitively impaired older persons with regards to the number of errors and/or reaction time. Effect sizes are located in the medium range (partial $\eta^{2}$ between 0.22 and 0.41), except for the number of errors in the selective attention task (partial $\eta^{2}=0.17$ ). The non-aggregated performance variables which differ mostly between the two groups are reaction times in the selective and divided attention test (partial $\eta^{2}=$ 0.38 and 0.41 , respectively). As a second step, the CI-Group and the young control group were compared and significant differences were found. The factor "group" accounts for $92 \%$ of the variance of the BCST total score, which indicates a strong effect. When comparing these two groups, medium to large differences are found for all subtests of the BCST (partial $\eta^{2}$ between 0.39 and 0.80). The largest group difference is found in reaction time to target stimuli in the divided attention task (partial $\eta^{2}=0.80$ ).

\subsection{Effect of Cognitive Status on Driving-Relevant Abilities}

In order to explore the isolated effect of cognitive status on performance in the BCST, a one-way
MANCOVA with cognitive status (impaired, normal) as between-subjects factor and age as covariate was calculated. Results showed that differences between cognitively normal and cognitively impaired subjects were still significant after controlling for age ( $p$-values from $<0.001$ to 0.007$)$ with effect sizes from 0.14 to 0.28 .

\subsection{Comparison of Performance under Single and} Dual Task Condition

To investigate the effects of dual tasking in the three groups, a mixed-model ANOVA with the factors group (CI-Group, older control group, young control group) and task condition (single task, dual task) was calculated for both subtasks of the divided attention test (Test 3), i.e., response to peripheral stimuli and steering. Results revealed significant global differences for both factors, that is between the groups (Pillai-Spur $=0.6, F(6,100)=7.6, p<0.001$, partial $\left.\eta^{2}=0.31\right)$ and between single and dual task condition (Pillai-Spur $=0.8, F(3,49)=71.6, p<0.001$, partial $\left.\eta^{2}=0.81\right)$. Furthermore, a significant interaction group $\times$ task condition was found (Pillai-Spur $=0.3$, $F(6,100)=2.7, p=0.016$, partial $\left.\eta^{2}=0.14\right)$, indicating that dual tasking affected performance of 
the three groups in different ways. To further clarify these results, each performance variable (i.e., errors in the two tasks and reaction time in the peripheral task) was analyzed with a separate group $\times$ condition ANOVA. Results show a significant effect of task condition (single task, dual task) on errors in the peripheral task $\left(F(1,51)=131.4, p<0.001\right.$, partial $\eta^{2}$ $=0.72)$ and reaction time to peripheral stimuli $(F(1$, $51)=6.2, p=0.016$, partial $\left.\eta^{2}=0.12\right)$, but not for errors in the steering task $(F(1,51)=0.10, p=0.76$, partial $\left.\eta^{2}<0.01\right)$. This finding indicates that all groups showed worse performance under dual task compared to single task condition in the peripheral task, while the steering task was unaffected by dual tasking (Fig. 2). Furthermore, a significant interaction group $\times$ task condition was found for errors in the peripheral task $\left(F(2,51)=6.5, p=0.003\right.$, partial $\left.\eta^{2}=0.20\right)$ but not in the steering task $\left(F(2,51)=0.2, p=0.495\right.$, partial $\eta^{2}$ $=0.03$ ). As can be seen in Fig. 2, this finding is due to the fact that the increase in errors going from the single to the dual task condition is larger for the CI-Group than for the other two groups. This is confirmed by a post-hoc analysis (Bonferroni correction), which yielded a significantly higher increase in errors in the peripheral task for the CI-Group than for the two other groups $(p<0.05)$.

\section{Discussion and Conclusions}

In the present study, the BCST [13] was used to assess driving-relevant functions (i.e., selective and divided attention, eye-hand-coordination, executive functions, and distance and speed regulation) in a group of cognitively impaired older subjects, a group of cognitively normal older subjects, and a group of young subjects. In order to control for age and/or gender, the groups were matched. The aim was to investigate the effects of cognitive impairment in older persons on driving-relevant skills and to explore age-independent differences between cognitively impaired and cognitively normal persons.

Results showed that cognitively impaired older persons performed significantly worse in all assessed skills than an age and gender-matched group of cognitively normal older subjects. Effect sizes suggest that, within a given test, reaction times are somewhat more sensitive to cognitive impairment than accuracy,

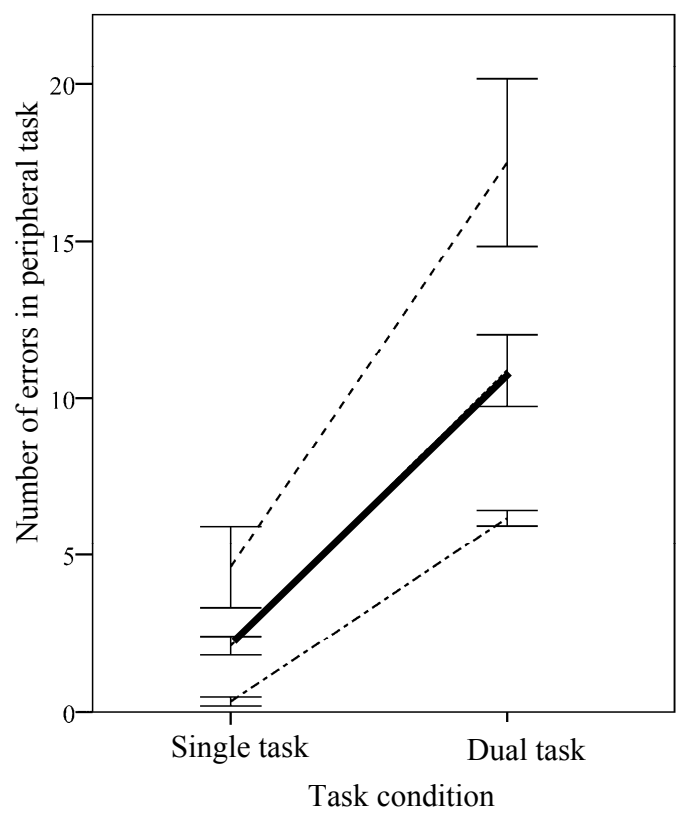

(a)

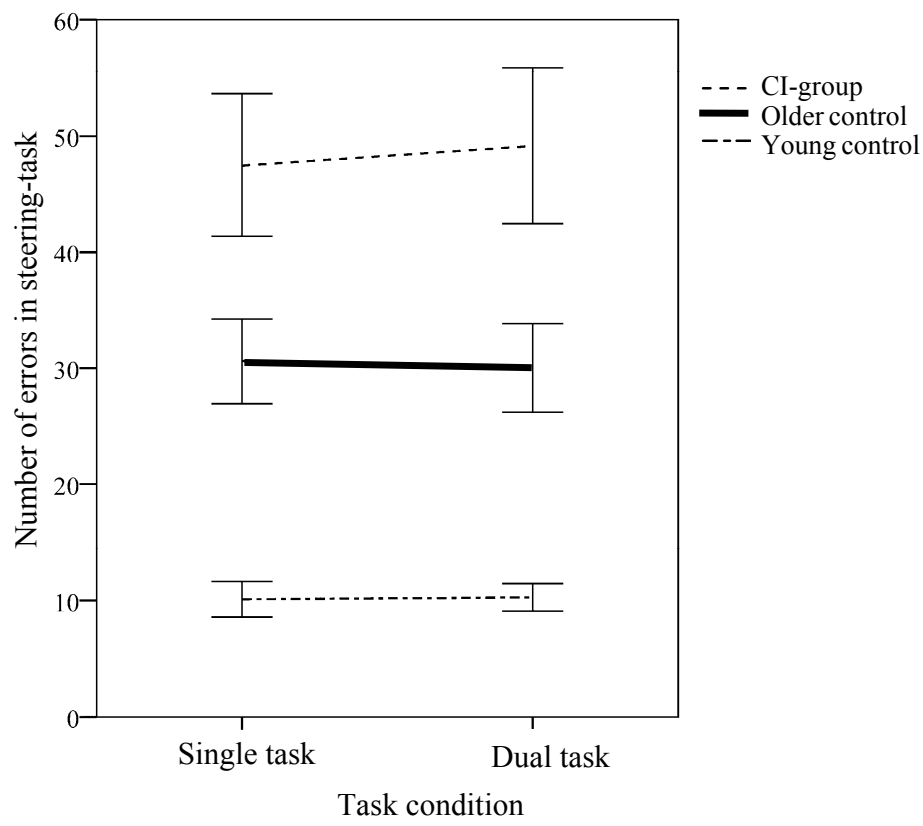

(b)

Fig. 2 Number of errors under single (Test 1) and dual (Test 3) task condition for the three groups in: (a) peripheral task; (b) steering task. Errors bars indicate standard errors. 
i.e., the number of committed errors. This is especially true for simpler tasks, such as the selective attention task in the BCST [13]. This finding is in line with previous studies, which showed that reaction times distinguish more precisely than accuracy between cognitively impaired and cognitively normal older adults [19, 20]. When comparing performance of cognitively impaired older subjects and young subjects, group differences are more accentuated with larger differences in all single skills. As in the comparison of the two older groups, highest effect sizes are found for differences in reaction times. After statistically controlling for age, cognitive status (impaired, normal) accounted for $14 \%$ to $28 \%$ of the variance in performance, highlighting the age-independent detrimental effect of cognitive impairment on driving-relevant cognitive abilities [2, 4]. The ability to perform multiple tasks at a time is fundamental for driving [6] and research indicates that this ability may be affected even in persons with very mild cognitive impairment [12].

In the present study, dual tasking (divided attention) consisted of performing in parallel a steering task and a peripheral selective attention task (i.e., responding to targets and ignoring distractors). All three groups performed less well under dual task than under single task conditions. This was also found in other studies [21]. More in detail, results show significantly worse performance under dual task condition for all groups in the peripheral selective attention task but not in the steering task. This is likely due to a prioritization of the steering task over the peripheral selective attention task, meaning that participants treated the steering task as primary task and allocated only available extra cognitive capacity to the peripheral task. It has to be noted that participants were instructed to perform both tasks at the same time with equal diligence, so the observed prioritization was spontaneous. A significant interaction group $\times$ condition and subsequent post-hoc analyses revealed that dual tasking affected performance in the peripheral task significantly more in cognitively impaired older subjects than in cognitively normal older subjects and young subjects. Given that the steering task was not negatively affected by dual tasking, it seems that cognitively impaired subjects treated the dual task condition almost as if it were a single task condition, focusing on one task (steering) while neglecting the other (peripheral task). This finding compares nicely to previous studies, which have shown that restricted cognitive capacity under dual task condition leads to a serialization of task performance, i.e., subjects focus on one task at a time and ignore the other [7, 8, 22].

The study has some limitations. First, a relatively small number of subjects was included. The etiology of cognitive impairment was not considered, leading to a relatively heterogeneous group of cognitively impaired older subjects. The aim of this study was to investigate how cognitive impairment affects driving-relevant skills in older adults and to quantify the extent and describe the pattern. Results show that cognitive impairment has a major, age-independent impact on driving-relevant cognitive skills in older persons. The relationship between driving-relevant cognitive performance, as assessed with the BCST, and behavior in real traffic in cognitively impaired persons is of major interest and can now be investigated as a next step.

\section{References}

[1] Sherman, F. T. 2006. "Driving the Ultimate IADL." Geriatrics 61 (10): 9-10.

[2] Drazkowski, J. F., and Sirven, J. I. 2011. "Driving and Neurologic Disorders.” Neurology 76: 44-9.

[3] Emerson, J. L., Johnson, A. M., Dawson, J. D., Uc, E. Y., Anderson, S. W., and Rizzo, M. 2012. "Predictors of Driving Outcomes in Advancing Age.” Psychol Aging 27: 550-9.

[4] Rizzo, M., and Kellison, I. L. 2010. "The Brain on the Road.” In Neuropsychology of Everyday Functioning, edited by Grant, I., and Marcotte, T. D. New York: Guilford Press.

[5] Whelihan, W. M., DiCarlo, M. A., and Paul, R. H. 2005. “The Relationship of Neuropsychological Functioning to Driving Competence in Older Persons with Early 
Cognitive Decline.” Arch Clin Neuropsych 20: 217-28.

[6] Chaparro, A., Wood, J. M., and Carberry, T. 2005. "Effects of Age and Auditory and Visual Dual-Tasks on Closed Road Driving Performance.” Optometry Vision Sci 82: 747-54.

[7] Andrews, E. C., and Westerman, S. J. 2012. “Age Differences in Simulated Driving Performance: Compensatory Processes.” Acc Anal Prev 45: 660-68.

[8] Blomqvist, L. H., Mynttinen, S., Backman, M., and Mikkonen, V. 1999. “Age-Related Differences in Driving: Are Older Drivers More Serial?” Int J Behav Dev 23: 575-89.

[9] Owsley, C., McGwin, G., and Ball, K. 1998. "Vision Impairment, Eye Disease, and Injurious Motor Vehicle Crashes in the Elderly.” Ophthalmic Epidemiol 5: 101-13.

[10] Anguera, J. A., Boccanfuso, J., Rintoul J. L., Al-Hashimi O., Faraji, F., Janowich, J., Kong, E., Larraburo, Y., Rolle, C. Johnston, E., and Gazzaley, A. 2013. "Video Game Training Enhances Cognitive Control in Older Adults.” Nature 501: 97-101.

[11] Hoffman, L., McDowd, J. M., Atchley, P., and Dubinsky, R. 2005. "The Role of Visual Attention in Predicting Driving Impairment in Older Adults.” Psychol Aging 20: 610-22.

[12] Dannhauser, T. M., Walker, Z., Stevens, T., Lee, L., Seal, M., and Shergill, S. S. 2005. "The Functional Anatomy of Divided Attention in Amnestic Mild Cognitive Impairment.” Brain 128: 1418-27.

[13] Bieri, R., Jäger, M., Gruber, N., Nef, T., Müri, R., and Mosimann, U. P. 2014. "A Novel Computer Test to Assess Driving-relevant Cognitive Functions-A Pilot Study.” International Psychogeriatr 26 (2): 229-38.
[14] Nasreddine, Z. S., Phillips, N. A., Bédirian, V., Charbonneau, S., Whitehead, V., Collin, I., Cummings, J. L., and Chertkow, H. 2005. "The Montreal Cognitive Assessment, MoCA: A Brief Screening Tool for Mild Cognitive Impairment." Journal of the American Geriatrics Society 53 (4): 695-9.

[15] Ricci, R., Cedrone, C., and Cerulli, L. 1998. "Standardized Measurement of Visual Acuity." Ophthalmic Epidemiol 5: 41-53.

[16] Reitan, R. M. 1992. Trail Making Test. Reitan Neuropsychology Laboratory.

[17] Shulman, K. I. 2000. "Clock-Drawing: Is It the Ideal Cognitive Screening Test?” Int J Geriatr Psych 15: 548-561.

[18] Cohen, J. 1988. Statistical Power Analysis for the Behavioral Sciences. L. Erlbaum Associates.

[19] Darby, D., Maruff, P., Collie, A., and McStephen, M. 2002. "Mild Cognitive Impairment Can Be Detected by Multiple Assessments in a Single Day." Neurology 59: 1042-46.

[20] Ritchie, K., Artero, S., and Touchon, J. 2001. "Classification Criteria for Mild Cognitive Impairment: A Population-Based Validation Study.” Neurology 56: 37-42.

[21] Ba, Y., and Zhang, W. 2011. "A Review of Driver Mental Workload in Driver-Vehicle-Environment System.” In Proceedings of 4th International Conference: Internationalization, Design and Global Development, Orlando, FL, USA, 125-34.

[22] Bélanger, A., Gagnon, S., and Yamin, S. 2010. “Capturing the Serial Nature of Older Drivers' Responses towards Challenging Events: A Simulator Study.” Acc Anal Prev 42: 809-17. 